\title{
Remote Supervision of Production Processes in the Food Industry
}

\author{
J. Lima, J. F. P. Moreira, R. M. Sousa \\ Department of Production and Systems Engineering, University of Minho, Guimarães, Portugal \\ (jorgeslima88@gmail.com; \{fmoreira, rms\}@dps.uminho.pt)
}

\begin{abstract}
This paper presents a remote monitoring system based on the SCADA model (Supervisory Control And Data Acquisition), specifically developed for automated production processes in a food company.

The goals defined for this project were the monitoring of three production lines and six silos of raw materials, along with the indication / evaluation of three performance measures demanded by the company: production rate, packed quantity and OEE (Overall Equipment Effectiveness). The developed system should also include other typical SCADA functionalities, namely alarms management, process trending and data logging. The applied methodology involved the detailed analysis of the existing automation systems, the functional specification of the remote monitoring system and the corresponding implementation (using the LabVIEW platform), test and validation. The project took about six months and the system was successfully implemented in one of the company's factories. All the objectives were achieved.
\end{abstract}

Keywords - OEE, production, SCADA, LabVIEW.

\section{INTRODUCTION}

To face the ever-growing competitiveness of the current markets, companies need to improve the performance of their production systems by taking advantage of all existing resources. Appropriate monitoring systems can be developed in order to acquire the data necessary not only to ensure the proper functioning of the production processes, but also to allow the analysis of the processes' behavior along a specific time period (trending), by computing a set of performance measures. This analysis can provide a significant input for the development of further improvements on the production processes, namely in terms of efficiency.

In the highly regulated food industry the data acquisition for monitoring production processes is of vital importance. It is extremely important to monitor the key variables involved in these processes, thus detecting any eventual problems that might endanger the compliance of the final product.

The main objective of this paper is to present a SCADA based system for remote monitoring of three production lines and six silos of raw materials in a company from the food industry, implemented with the LabVIEW development platform. The LabVIEW's DSC

This work was partly financed by the Portuguese Foundation for Science and Technology, project Pest-OE/EME/UI0252/2014.
(Datalogging and Supervisory Control) module is explored in order to assess its adequacy for the project. Besides the monitoring of several process variables (e.g. temperature, pressure, weight in process and stirring speed), the intended functionalities include: alarms management, process trending and data logging. Furthermore, three performance indicators were specifically requested by the company: production rate, packed weight and OEE.

The paper is structured in five sections. After this introduction, section 2 presents a brief literature review on the main concepts and tools used in the project. The third section describes the system design and implementation. Discussion is presented on section 4 , and on section 5 the final remarks are outlined.

\section{LITERATURE REVIEW}

Manufacturing processes in the food industry are usually hard to automate due to their non-uniformity, high variability of raw materials and also to the scarcity of sensors for real-time monitoring of the key variables. Thus, the introduction of computer-aided systems in this kind of industry has been slower than in other process industries [1]. However, the need to remain competitive in today's turbulent markets led the companies to invest in the implementation of automated production systems. In fact, a major benefit of automation and process control is the ability to produce a given range of products in a consistent manner [2], providing the most adequate software and hardware solutions that can be modified and upgraded according to the company needs [3].

According to [4], SCADA systems are especially adequate for the monitoring of geographically distributed manufacturing systems. Although they can also perform some control, in practice they are mostly used as supervision systems [5]. Fig. 1 depicts a typical SCADA architecture with three layers.

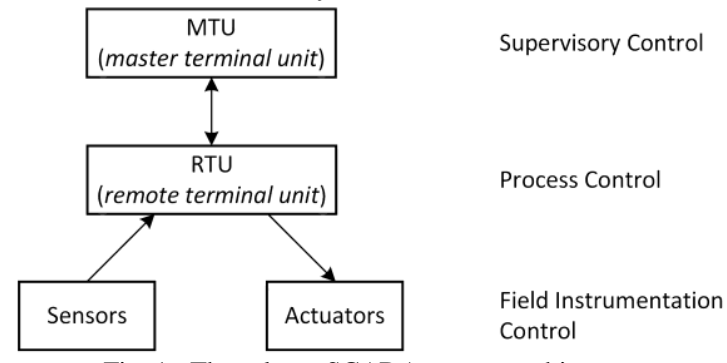

Fig. 1. Three-layer SCADA system architecture. 
The sensors and actuators constitute the lower level of the hierarchy, i.e. the field instrumentation control layer, being responsible for the acquisition of input signals and delivery of control ones to the processes [6].

The process control layer is composed by RTUs (Remote Terminal Units), usually PLCs (Programmable Logic Controllers). However, SCADA systems can also be used with local equipment (factory-level supervision) or with both local and remote equipment [4].

The PLCs are robust devices, specially designed to withstand the adverse conditions that often occur in the industry. They are usually composed by: power supply, microprocessor, input/output module (analog and/or digital) and communications module. PLCs can be programmed to execute a set of functions (arithmetic, logic, control, monitoring, etc.) so they can take full control of industrial machines and processes [7]. On the process control layer it is also quite common the use of HMIs (Human-Machine Interfaces), i.e. terminals that allow workers to check the state of the processes.

The supervision control layer is implemented by the MTU (Master Terminal Unit), typically a computer (e.g. a personal computer), that periodically collects data from the process control layer. The MTU ensures essential functionalities such as alarms management, data logging, processes trending and reports generation, being thus an important tool for the companies' management.

Several indicators can be used to monitor the performance of a production process. The number and type of adopted performance measures depends not only on the nature of the business, but also on the strategy set by the companies to cope with the competitive environment in which they operate. According to [8] some typical examples are: (i) production rate (e.g. in parts/h), (ii) utilization rate (ratio between produced quantity and productive capacity), (iii) lead time (time period necessary to deliver a product), (iv) work-inprocess (WIP) and (v) Overall Equipment Effectiveness (OEE). OEE is widely adopted by many companies from different areas and indicates how effectively their equipment is used [9]. Furthermore, the OEE determination process shows which aspects must be intervened in order to improve the referred effectiveness. The OEE is determined by the following equation:

$$
\mathrm{OEE}=\text { Availability } \mathrm{x} \text { Performance } \mathrm{x} \text { Quality }
$$

The availability is defined as the ratio between the real operation hours (shift time minus the planned stops minus the unplanned stops) and the expected operation hours (shift time minus the planned stops). Performance is obtained dividing the average production rate by the best production rate ever attained. The quality factor is the quotient between the quantity of good products and the total quantity of products.

LabVIEW - Laboratory Virtual Instrument Engineering Workbench from National Instruments Co. is a graphical programming platform that has been frequently used to develop industrial monitoring systems.
LabVIEW has its roots in automation control and data acquisition. Its graphical representation, similar to a process flow diagram, was created to provide an intuitive programming environment to scientists and engineers. It has several important features that include simple network communication, connection through common protocols (RS232, GPIB, etc.), a powerful toolset for process control and data fitting, fast and simple user interface development and an efficient code execution environment [10]. To develop SCADA applications, which include the communication with PLCs, LabVIEW provides the DSC module (Datalogging and Supervisory Control). This module allows the development of distributed monitoring systems with thousands of variables, recorded in Citadel database. Some examples of SCADA based projects implemented with LabVIEW can be found in $[2,3,11]$.

\section{SYSTEM DESIGN AND IMPLEMENTATION}

The system architecture is depicted in Fig. 2. The SCADA application uses client-server communication. The PLCs acquire the process data on each production line as well as the signals from the sensors installed on all the raw material silos. The PLCs are linked via Ethernet to the server application, which records the data on a MySQL database (DB) and the alarms on the Citadel DB. The system can be configured with multiple servers for redundancy purposes. Therefore, on an eventual problem with one server, others would be available.

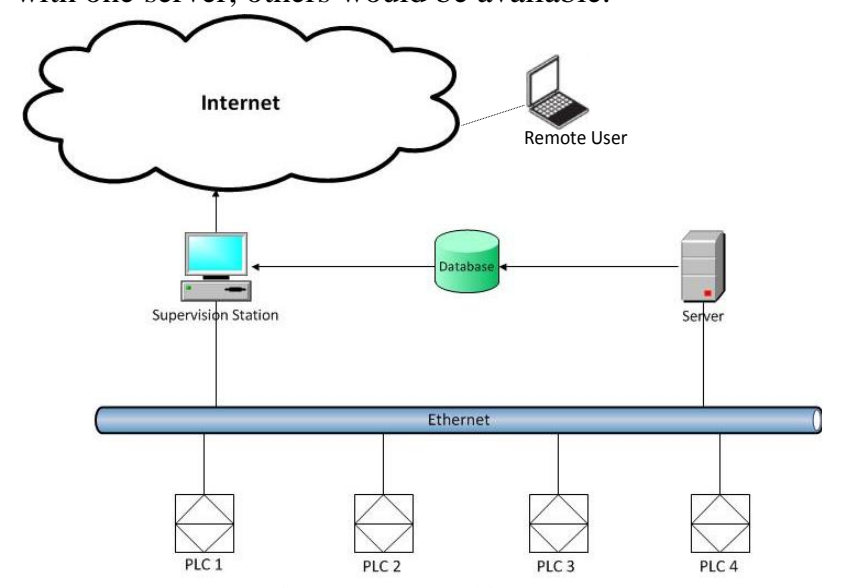

Fig. 2. System architecture.

Specific LabVIEW applications were developed for the Server, the Supervision Station, and the Remote User access. The LabVIEW applications (named Virtual Instruments-VI) comprise a front panel, i.e. the user interface, and a block diagram, representing the program code, on the $G$ graphical language. The LabVIEW Database Connectivity Toolkit supports the use of SQL databases. This toolkit has a full range of SQL functions and allows ODBC connections with SQL databases. The Citadel DB was used on this project for datalogging of the production processes and to register the alarms, as well as the MySQL DB for registering the data of three production lines. 


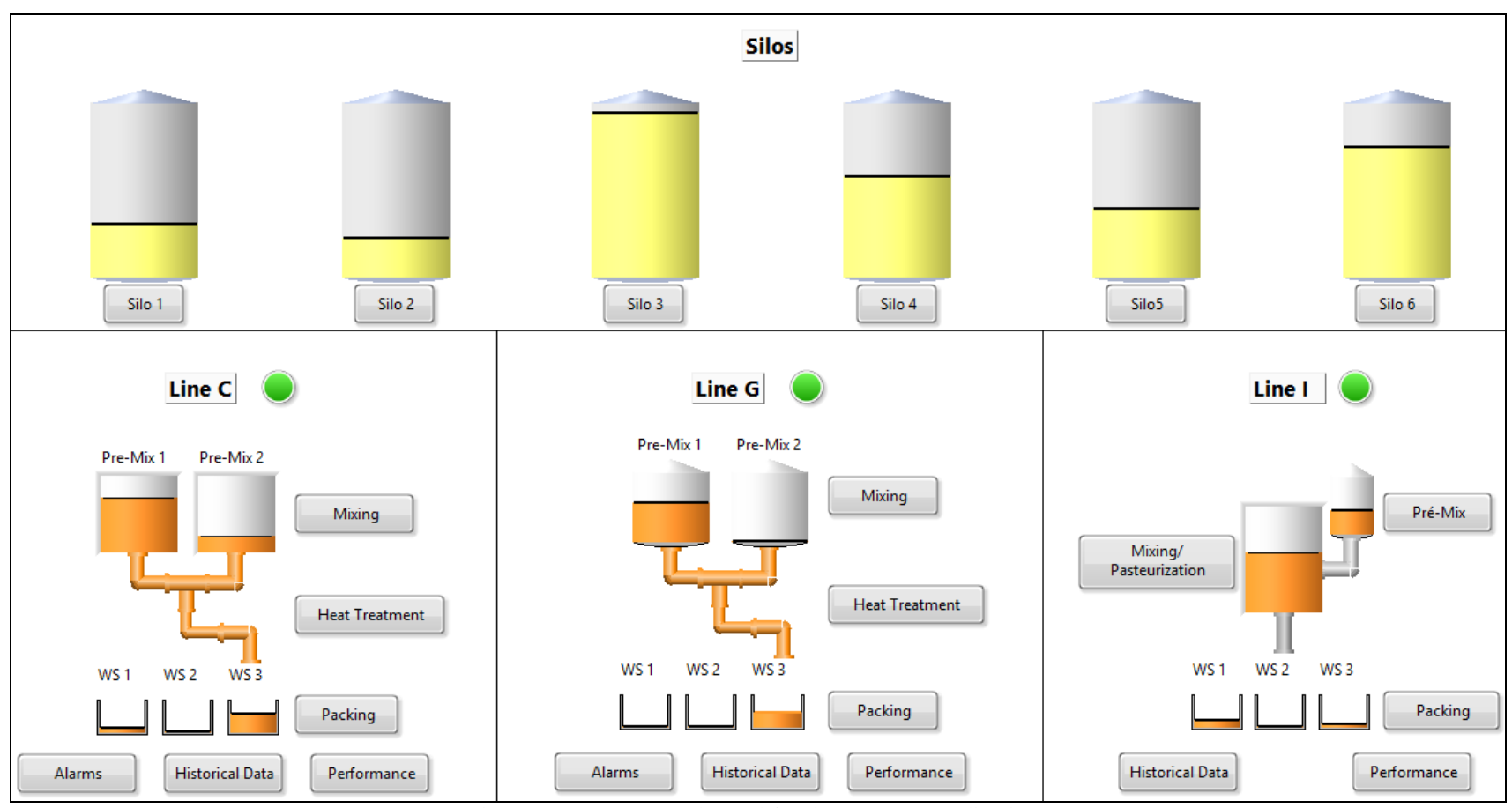

Fig. 3. HMI VI Control Panel for the SCADA application.

The remote access to the supervision application was enabled using LabVIEW remote panels.

The main VI (running on the supervision station) front panel, illustrated on Fig. 3, globally represents the system in real time. The top part displays the status of the silos and the bottom part the three production lines (lines C, G and I).

Each production line consists of a number of operations executed in three workstations (WS1 to WS3). Alarms monitoring, access to historical data and performance indicators are available for all three production lines. A number of subVIs is invoked from within the main VI, each of which specifically addressing single production processes or functionalities.

\section{A. PLC Connection}

The communication with the PLCs is made via OPC (Object Linking and Embedding for Process Control). The LabVIEW DSC module includes an OPC server whose connection to PLCs is possible to configure. The tags of the OPC server were configured to index each position of the PLC memory, where the data from the sensors is recorded. The rate of access to this data was configured to $1000 \mathrm{~ms}$ and the access type was also configured. The option Create Bound Variables, enabled the creation of shared variables of the tags. Therefore access to the sensors data simply requires an access to the respective shared variable.

\section{B. Datalogging}

On a monitor application it is required to register the data collected on the production processes for further future analysis. This process is designated datalogging and can be performed in LabVIEW by using the Citadel
DB which is included on the DSC module. The Citadel DB records all historical data from the monitored variables, after activation of the respective functionality on the properties of the shared variables. Alternatively, this can be done programmatically using the function configure logging. The values are not saved on the DB periodically, instead, only a new value is saved, if a predefined variation on its value is verified. The configuration parameters are: (1) the value resolution which defines the precision of the value, and (2) the value deadband - which defines the required percentage variation that qualifies for DB value update. Adequate adjustment of these parameters is required to avoid excess or insufficiency of data on the processes.

Processes data logging, registering the processes specifics on each phase on the production lines is made possible using the relational database MySQL. Three tables were created, one for each production line, which record the processID, designation, production order and the start and end time stamps. The data relating the packaging processes are also registered on the DB, namely the processID, weight and packing time.

The specific monitoring for a given production line can be accessed by clicking on the corresponding button on the main HMI (Fig. 3), e.g. the Mixing process for Line $\mathrm{C}$ is depicted on Fig. 4. Here, real time data is provided on the pre-mix 1 and pre-mix 2 processes (the green LEDs on the top of the panel indicate when the processes are running): (1) process ID, (2) production order, (3) temperature, (4) weight and (5) stirring speed. Additional information on this process can be obtained by clicking on the Process button. Historical data on this process is also available for consultation by clicking on the Graphs button. 


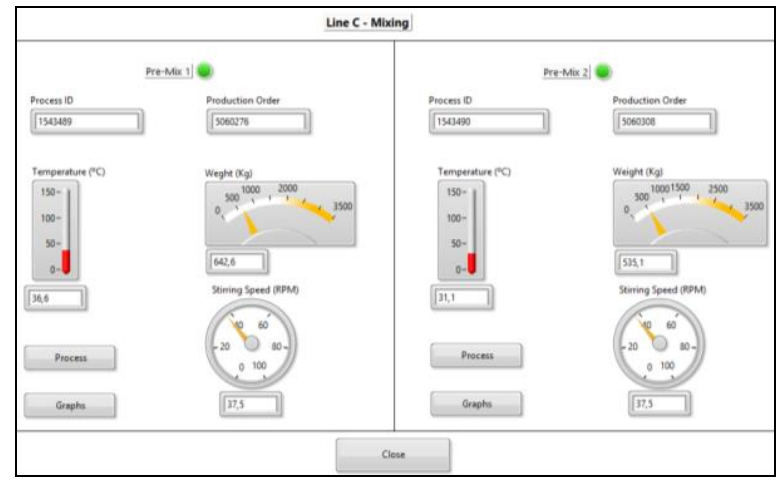

Fig. 4. Process monitoring at Line C.

\section{Trending}

The trending functionality, available in LabVIEW under the Historical Trend function, enables the access to the Citadel DB of a given process, and particular process stages, over a given timeframe. This provides a graph of the historical evolution of the relevant shared variables within the specified timeframe, or, otherwise, the real time data from the shared variables, beginning with data from the start of the day. This functionality enables the detection of trends on the data (variables) which might indicate possible abnormal situations at short to middle term, therefore enabling for prior precautionary actions from the line operators or site managers.

\section{Alarms}

A number of critical process variables were identified, e.g. the temperature of the heat treatment should comply with temperature limits. LabVIEW enables the activation of logging and alarming functionalities by configuring the properties of shared variables. This enables the registration of variable values on the Citadel DB, the triggering of visual alarms on the application HMI, among others. Fig. 5 illustrates the occurrence of one such alarm on line G. Here, this event triggers a visual alarm (the pipe changes from grey to red and the heat treatment button flashes red).

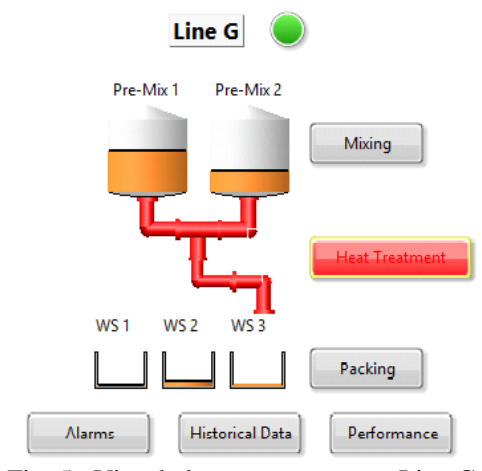

Fig. 5. Visual alarm occurrence at Line G.

Alarm registration and subsequent data analysis is quite an important issue, bearing in mind that food preparations for human consumption is a highly regulated industry, with absolute safety assurance requisites and demanding requirements on quality and traceability, thus being key enablers to the success of the business.

\section{E. Key Performance Indicators}

Three KPIs were considered on each line: (1) packed weight; (2) production rate; and (3) OEE. By clicking on the Performance button on the main front panel for each production line (Fig. 3) a new window opens, enabling the access to each one of the KPIs, as depicted on Fig. 6. The user selects the Type of Analysis, Start Date and End Date, following by a selection of the specific KPI. A graph shows the evolution of the selected KPI over the specified timeframe, and the KPI current value. When Packed Quantity is selected, the respective quantity is shown and contrasted with the ideal one, i.e. the max quantity that the system is capable of. When Output Rate is selected, the registered values for that rate are shown as well as the ideal rate for that line. When OEE is selected the percentage values are shown, as well as the Availability (\%) and Efficiency (\%) rates. Relating the quality rate, the company did not want to expose the exact figure and suggested that the value 1 should be used.

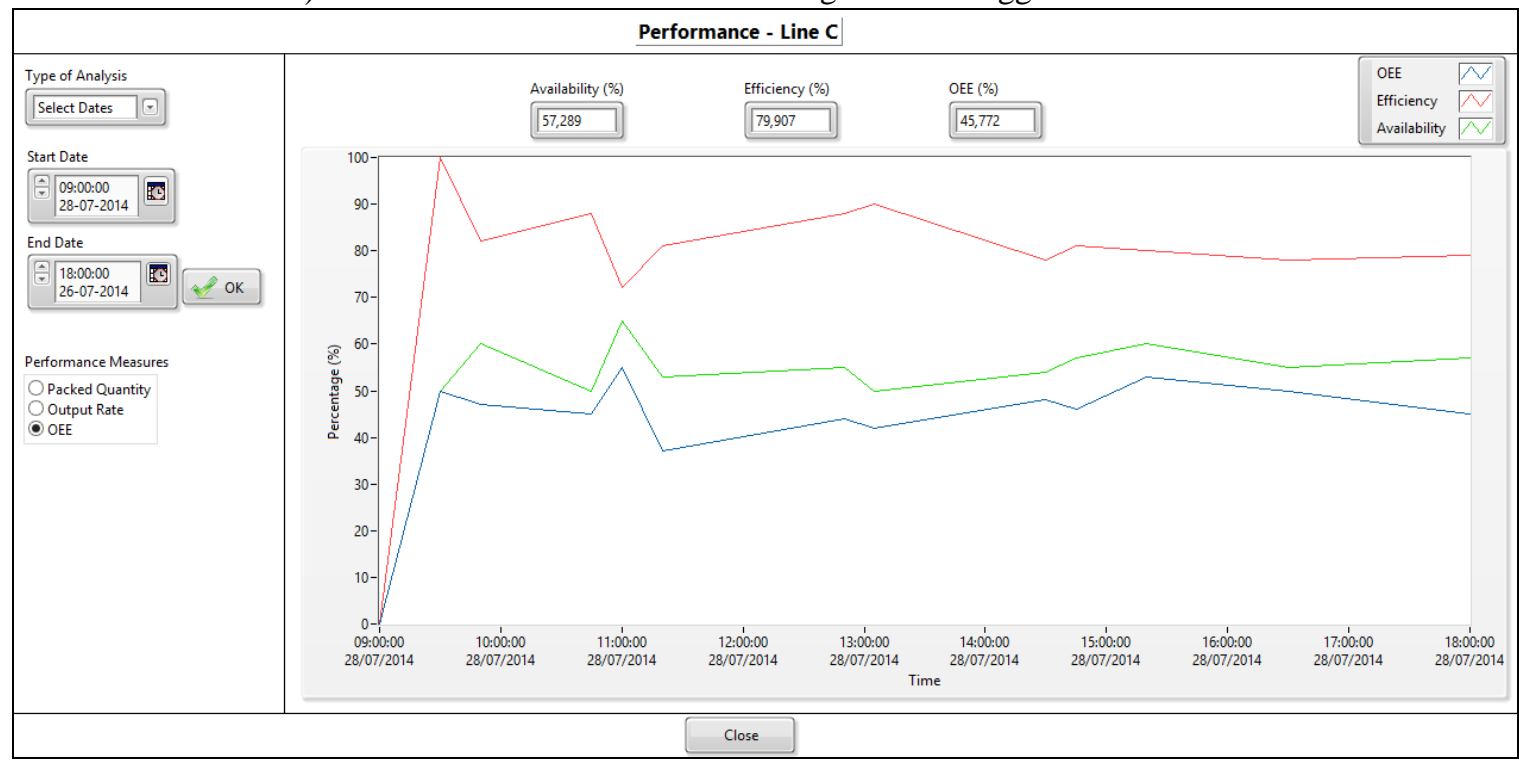

Fig. 6. HMI for the OEE KPI on Line C. 


\section{F. Web Version}

A web version of the supervision application was developed aiming the access from multiple remote users, using a web browser. This is supported by the LabVIEW remote panels functionality (installed on the client side), which consists on adapted VIs for integration on HTML pages. The remote panels operate in three modes: (1) embedded; (2) snapshot, and, (3) monitor. Mode (1) enables the users to remotely control the front panel, mode (2) enables to observe a static snapshot of the front panel, and mode (3) does not enable any possibility of control while continually updates images of the front panel. All three modes were made possible on the web version of the application.

\section{DISCUSSION}

The developed SCADA system was acknowledge by the company to consistently produce reliable key data on the system, in real-time, remotely and concurrently with other similar applications. New functionalities were deployed in order to provide the control of the HMI over a web browser, thus removing geographical barriers on accessing shopfloor operational data. The developers considered the architecture deployed and the LabVIEW development tool adequate for the application purpose.

The system can be readily expanded so as to incorporate many more production lines and raw materials silos on the same plant, since it basically requires access to shared variables which are fed by a number of sensors attached to PLCs. Furthermore, the approach taken, could be used to replicate similar factories and could be integrated into a unique HMI, or easily adapted to other types of industries, granted that the architecture remained pretty much similar.

The real-time data from the processes can be contrasted with historical data, and mismatches can be used has triggers for rapid action at shopfloor level, in order to avoid the strongest negative impacts, e.g. avoid severe food waste generation due to faulty or relaxed process control. This prospectively translates as well into improved effectiveness on the production system.

\section{CONCLUSION}

It is explained the design and deployment of a remote SCADA system on a facility of a major player of the food industry. The use of Key Performance Indicators was enacted and its rationale explained bearing in mind the field of application and its relevance on the business. It was also explained how the monitoring system could be expanded or replicated to other location facilities, how it could be integrated on the existing system and the estimated effort on doing so.

Comparison with other solutions was attempted but not brought to an ideal state worth mentioning.
The SCADA systems hold a robust architecture that qualify for a progressive expansion, while providing multiple-level timely data, which enable the realization of value-added activities and relevant decision making across the organization, i.e. tactical and operational levels. The design principles, the selected development toolkit and the application specifics, can be feasibly applied to other production facilities, which require the remote control and monitoring of production processes.

\section{REFERENCES}

[1] S. Linko, and P. Linko, "Developments in Monitoring and Control of Food Processes," Food and Bioproducts Processing, 76, pp. 127-137, 1998.

[2] M. J. McGrath, J. F. O'Connor, and S. Cummins, "Implementing a Process Control Strategy for the Food Processing Industry," Journal of Food Engineering, 35, pp. 313-321, 1998.

[3] M. Endi, Y. Z. Elhalwagy, and A. Hashad, "Three-Layer PLC/SCADA System Architecture in Process Automation and Data," in Proc. The 2nd International Conference on Computer and Automation Engineering, ICCAE 2010, Singapore, pp. 774-779.

[4] B. Galloway, and G. P. Hancke, "Introduction to Industrial Control Networks," IEEE Communications Surveys \& Tutorials, vol. 15, no. 2, pp. 860-880, second quarter 2013.

[5] A. Daneels, and W. Salter, "What is SCADA?," in Proc. International Conference on Accelerator and Large Experimental Physics Control Systems, ICALEPCS 1999, Trieste, pp. 339-343.

[6] F. Moreira, and R. Sousa, Automatic Production Systems (in Portuguese), pedagogical publication, University of Minho, 2013.

[7] M. P. Groover, Fundamentals of Modern Manufacturing. John Wiley \& Sons, Inc., 2002.

[8] M. P. Groover, Automation, Production Systems, and Computer-Integrated Manufacturing. New Jersey: PrenticeHall, 2001

[9] M. M. Ahmad, and N. Dhafr, "Establishing and improving manufacturing performance measures," Robotics and Computer-Integrated Manufacturing, vol. 18, no. 3, pp. 171-176., 2002.

[10] C. Elliott, V. Vijayakumar, W. Zink, and R. Hansen, "National Instruments LabVIEW: A Programming Environment for Laboratory Automation and Measurement," Journal of Laboratory Automation, vol. 12, no. 1, pp. 17-24, 2007.

[11] S. Padhee, and Y. Singh, "Data Logging and Supervisory Control of Process using LabVIEW," IEEE Students' Technology Symposium, TechSym 2011, Kharagpur, pp. 329-334. 